\title{
High Repetition Laser Shock Peening on Magnesium Based Biodegradable
}

\author{
Alloys \\ Hossein Kamkarrad ${ }^{*}$, Sivakumar Narayanswamy ${ }^{* *}$ and Mohammad Keshmiri" \\ "Department of Mechanical Engineering, Concordia University. 1515 St. Catherine West. Montreal, Quebec H3G 2W1 Canada \\ h-kamkar@encs.concordia.ca\&mohammad.keshmiri@gmail.com \\ "* Associate Professor and M. Eng \& M.A.Sc. Program Director, Mechanical and Industrial Engineering, Concordia University, \\ Montreal, Canada nrskumar@encs.concordia.ca Tel: (514) 848-2424 (Ext.7923)
}

\begin{abstract}
New generation of metallic implants through capability of absorption within biological environment has been introduced in recent researches. Although, magnesium implant with proper biocompatibility has been proposed as biodegradable metallic implant, its insufficient mechanical attributes must be enhanced for implantation within human body. Laser shock peening has been successfully conducted for modification of magnesium implant. Overall, laser shock peening can be performed by low repetition rate (less than $30 \mathrm{~Hz}$ ) and high power pulsed laser which needs expensive devices. In order to laser shock peen magnesium alloys that have a lower mechanical strength, it is better to use high repetition pulsed laser which is much faster and economical. Recently, high repetition rate laser shock peening of magnesium has been theoretically found feasible in previous research of authors. In order to validate occurrence of high repetition rate laser shock peening, changes in surface topography and hardness are evaluated on the magnesium specimens.
\end{abstract}

DOI: 10.2961/jlmn.2015.03.0010

Keywords: Magnesium-High repetition rate laser shock peening-Biodegradable-RoughnessHardness

\section{Introduction}

Complications of second surgery for removal of permanent implants were the original idea to propose a new generation of biodegradable implants. Recently, magnesium based alloys have been identified as a potential degradable alloys [1]. These biomaterials could be excellent materials in terms of biomechanical aspects, have comparable mechanical strength to bones and have privilege of avoiding stress shielding [2]. However, in order to fix broken bone, fixators having sufficient mechanical strength are required. Otherwise, subsequent breakage of the treated bone can occur [3]. Increasing compressive residual stress (CRS) is the advanced methods that has been introduced for enhancement of mechanical attributes. Due to numerous exclusive merits, laser shock peening (LSP) has been recommended as a potential method for creation of CRS upon the specimen surface. Deeper and higher magnitude of CRS achieved with LSP [4] has desired performance of specimen in corrosive environment [5]. In addition, LSP has undeniable positive effect on fatigue strength of peened sample [6]. Currently, LSP is performed with high power low repetition pulsed laser [5-6,7]. High cost devices and low speed method are the main restrictions in LSP. Hence, it is appropriate to evaluate the possibility of high repetition laser shock peening (HRLSP) that is more economical and much faster as an alternative to LSP. For HRLSP, tight focusing of laser beam diameter to few micrometers, using appropriate confining medium, utilization of suitable protective coating and highly accurate adjustment of laser scan speed by a Galvo-mirror are proposed works in this manuscript. The fact that mechanisms of LSP and HRLSP has been based on the identical principles, similar to LSP the occurrence of HRLSP is recognized by a number of experimental methods such as measurement of changes in surface roughness, peening depth and hardness.

\section{High Repetition Laser Shock Peening (HRLSP)}

As long as the absorption of laser energy exceeds the material threshold, ablation is conducted and the material is transformed from solid to gas state, directly. The metallic gas captures adequate energy and its temperature increases drastically. Consequently, free electrons can be departed atoms and plasma is affected [7]. Plasma can generate a high pressure (GPa range) over the course of few nanoseconds. The achieved shock pressure can be calculated by the equations 1-4 [8].

$$
\begin{aligned}
& P=0.01 \sqrt{\frac{\alpha \cdot Z I}{2 \alpha+3}} \\
& \frac{2}{Z}=\frac{1}{Z s}+\frac{1}{Z c} \\
& I=\frac{P_{w} / f}{0.78 D^{2} \cdot \tau}=E / \tau A \\
& Z=\rho U
\end{aligned}
$$

Where, $\mathrm{P}(\mathrm{GPa})$ is the shock pressure, $\mathrm{Z}\left(\mathrm{g} / \mathrm{cm}^{2} . \mathrm{s}\right)$ is the impedance of shock wave, E (J) is average energy of a pulse, $\tau$ (nanosecond) is laser pulse duration and $\mathrm{D}(\mathrm{mm})$ is the diameter of laser beam or spot size, $\alpha$ is a constant that is proportional to efficiency of laser absorption (if most of laser 
energy absorbed by surface, $\alpha$ will be close to 0.1 ), $\mathrm{A}$ is spot size area, $I$ is laser intensity in $\mathrm{Gw} / \mathrm{cm}^{2}, P_{w}$ is average laser power in watt, $f$ is repetition rate in $\mathrm{Hz}$ and $\mathrm{Z}_{\mathrm{C}}$ and $\mathrm{Z}_{\mathrm{S}}$ are the impedances of confining medium and specimen, respectively. $\rho$ is density in $\mathrm{g} / \mathrm{cm}^{3}$ and $U$ is velocity of shock wave and it is equal to sound velocity in $\mathrm{cm} / \mathrm{s}$ within the material. Providing the shock load exceeds the Hugonoit Elastic Limit (HEL) [9], laser shock peening will be effected. HEL is presented by equation 5

$H E L=\frac{(1-\vartheta) \sigma_{Y}^{D y n}}{1-2 \vartheta}$

where $v$ is Poisson's ratio and $\sigma_{Y}^{D y n}$ is dynamic yield stress. Therefore, the principal term for laser peening is $P>H E L$, provided that pulse duration is adequately small and it should be proved by equilibrium in equation 6 [10].

$\tau \ll r_{0} \sqrt{\frac{\rho(\lambda+2 \mu)}{4 \mu(\lambda+\mu)}}$

Where, $\mu$ and $\lambda$ are lama constants, $r_{0}$ is radius of spot size and $\tau$ is pulse duration.

Equations 1 to 5 can reveal reasons of difficulty in HRLSP. Owing to $\mathrm{P}_{\mathrm{w}}$ and $\tau$ being approximately constant, as long as $f$ increases up to $10000 \mathrm{~Hz}$ in equation 3 , the intensity (I) decreases 10000 times. Hence, $\mathrm{P}$ in equation 1 decreases drastically and required pressure by equation 5 cannot be provided. In order to amplify shock pressure, reduction of beam diameter (D) in equation 3 and enhancing of $Z$ in equation 1 are two possible approaches. The $\mathrm{D}$ can be condensed by appropriate lens and $\mathrm{Z}$ can be enhanced by choice of proper confining medium (CM) and protective coating (PC). The equations 2 and 4 indicate impact of $\mathrm{CM}$ and $\mathrm{PC}$ on $\mathrm{Z}$ magnitude.

\subsection{Design of laser scanning system/specifications}

In order to avoid multiple laser shots at the same point, the scan speed should be designed, properly. The required peening scan speed $\left(\mathrm{S}_{\mathrm{s}}\right)$ without overlap along the $\mathrm{y}$-axis, is achieved by equation 7 .

$S_{s}=D \times\left[1+\left(1-O_{p}\right)(f-1)\right]$

Where, $\mathrm{O}_{\mathrm{p}}$ is percentage of overlap as displayed in figure 1 . From the equation 1-5, it can state the $40 \mu \mathrm{m}$ is an appropriate size of $\mathrm{D}$ for peening of magnesium specimens

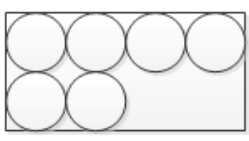

a) $0 \%$ overlaps

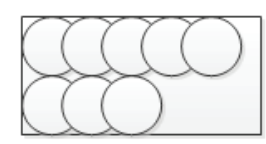

b) $33 \%$ overlap

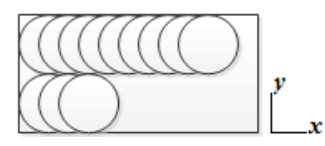

c) $66 \%$ overlap

Fig.1: Overlap along the $\mathrm{X}$-axis

where, repetition rate is $10 \mathrm{KHz}$. The speed calculation of the peening at $40 \mu \mathrm{m}$ spot size is explained in the next section. The proper scan speed at $40 \mu \mathrm{m}$ with $0 \%$ overlap is 400 $\mathrm{mm} / \mathrm{s}$. In order to, provide this high scan speed for LSP, use of galvo-mirror THORLABS GVSO02, is proposed. The system consists of two galvano mirrors each attached to a servo DC motor. The servo motors are controlled with a NI6211 USB DAQ board. This board gets the motor's desired position as an analog voltage and sets the motor's position to an angle proportional to the applied voltage. The range of the analog voltage is between -10 to +10 . The range of motion of

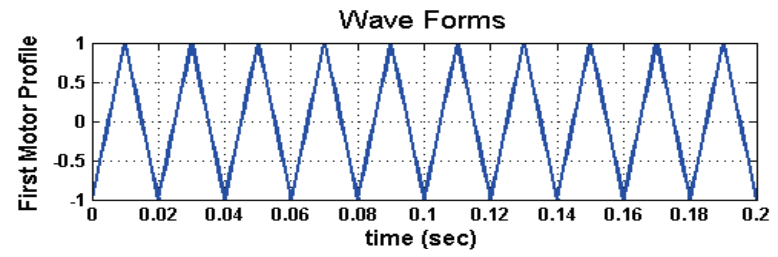

(a

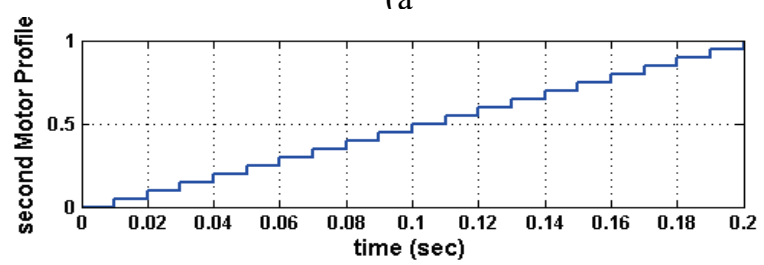

(b

Fig.2: DAQ signals for $\mathrm{X}$ and $\mathrm{Y}$ axis servo-motors

each motor is between $-12^{\circ}$ to $+12^{\circ}$. Hence, the voltage range

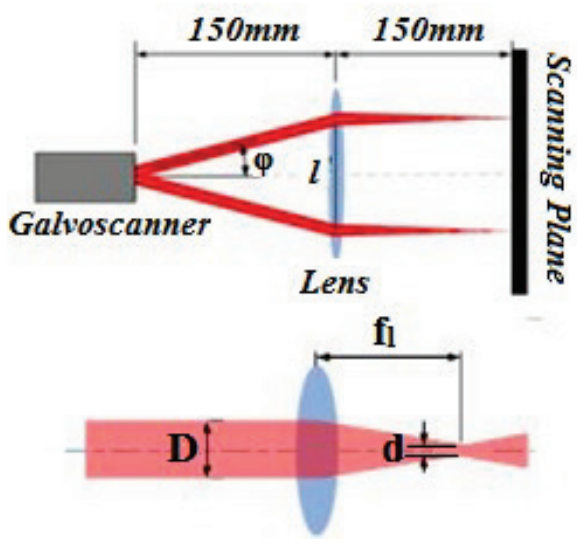

Fig.3: Adjustment optical devices for HRLSP

between -10 to +10 volts corresponds to $24^{\circ}$ of motion range which provide a resolution $1.2^{\circ} / \mathrm{V}$. The DAQ is equipped with two analog outputs which provide the desired set points for motors. A LabView program has been developed to control the output voltage and subsequently the motor position. Each motor is responsible for

1 degree of freedom (DOF) movement of the laser on the work piece. A desired path is produced to cover evenly a 10 $\mathrm{mm}$ square on the work piece. For this, one of the motor is controlled using a triangular profile signal (Figure 2-a) with a magnitude that covers the $10 \mathrm{~mm}$ in the $\mathrm{X}$ axis. At each top and bottom peak of the triangular profile the other motor moves forward one step in the $\mathrm{Y}$ axis and remains at that 
position until the next peak of the triangular profile (Figure 2-b) covering the square of $10 \mathrm{~mm}$ on the workpiece.

The laser that is used in this work is PRISMA TM1064-V diode pumped, solid-state laser and its specifications are given in table 1. Aforementioned, the scan speed at $40 \mu \mathrm{m}$ with $0 \%$ overlap is $400 \mathrm{~mm} / \mathrm{s}$. From figure $3, l=10$ this means $400 / 10=40$ lines need to be scanned in a second. In other words, mirror oscillation frequency (MOF) should be $40 \mathrm{~Hz}$. For spot size of $60 \mu \mathrm{m}$, the required scan speed is $600 \mathrm{~mm} / \mathrm{s}$ according to equation 7 . Therefore, MOF must be $60 \mathrm{~Hz}$. Determination of spot diameter (d) is conducted by equation 1 and 3 for having a successful HRLSP. Spot size is a function of initial laser beam diameter (D), laser wavelength $(\lambda)$ and focal length $\left(f_{L}\right)$ of the lens. By use of equation 8 , the required initial beam diameter has been determined in table 1 . $d=1.27 \times f_{l} \frac{\lambda}{\mathrm{D}}$ 8

Table.1: Laser specifications for 40 and $60 \mu \mathrm{m}$ spot sizes

\begin{tabular}{lllccc}
\hline $\mathbf{d}^{*}$ & $\mathbf{D}^{*}$ & $\mathbf{M O F}_{\mathbf{0}}{ }^{*}$ & $\mathbf{M O F}_{\mathbf{3 3}}$ & $\mathbf{M O F}_{66}$ & \\
\hline 40 & 5.06 & 30 & 19.8 & 9.9 & \\
60 & 3.37 & 60 & 39.6 & 21.78 & \\
\hline $\mathbf{d}^{*}$ & $\boldsymbol{\Phi}_{\boldsymbol{X}}{ }^{\circ}$ & $\boldsymbol{\Phi}_{\mathbf{Y}}{ }^{\circ}$ & $\mathbf{S}_{\mathbf{0}}{ }^{*}$ & $\mathbf{S}_{\mathbf{3 3}}$ & $\mathbf{S}_{\mathbf{6 6}}$ \\
\hline 40 & \pm 1.909 & 0.015 & 400 & 266.66 & 133.33 \\
60 & \pm 1.909 & 0.022 & 600 & 400 & 200 \\
\hline
\end{tabular}

\section{d: Spot size ( $\mathrm{mm}) \quad$ D: Initial beam diameter ( $\mathrm{mm})$ MOF: Mirrors Oscillation Frequency $(\mathrm{Hz})$ \\ Ф: Mirrors Oscillation S: Scan speed $(\mathrm{mm} / \mathrm{s})$ \\ The subscript 0,33 and 66 define the \% overlap as in}

\subsection{Peening specifications:}

Excluding of effectivness of laser specifications on HRLSP, peening parameters such as strength of specimen, confining medium (CM) and protective coating (PC) have fundamental influence on occurance of HRLSP.

The equations 2 and 4 define impact of $\mathrm{CM}$ on shock pressure. Quartz and glass with higher impedance ( $\mathrm{Z}$ )

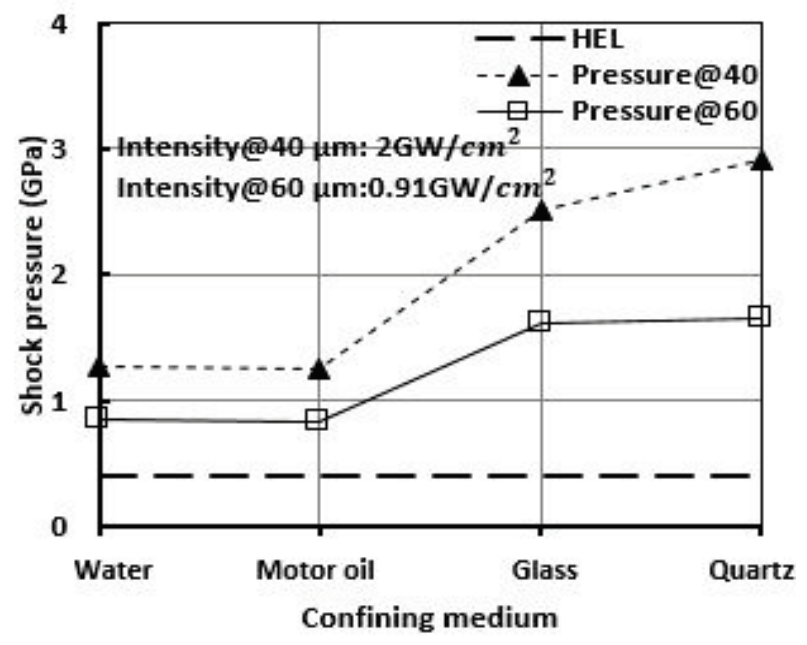

Fig.4: Impact of confining medium (CM) on shock pressure

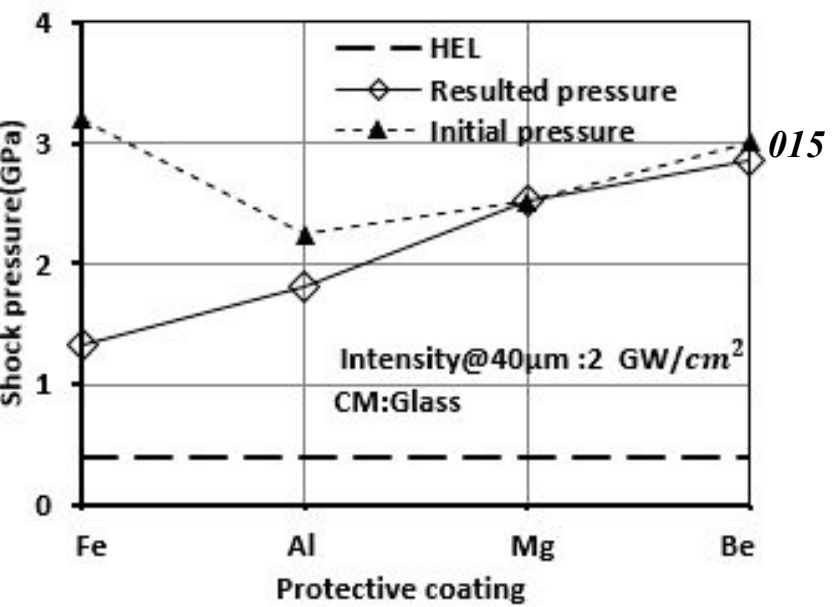

Fig.5: Impact of protective coating (PC) on shock pressure

compared with air, water and motor oil can increase $Z_{c}$. Increasing of $Z_{c}$ leads raising $Z$ and enhancement of shock pressure has been resulted. The figure 4 demonstrate significance of $\mathrm{CM}$ on enhancement of shock pressure, where applying of quartz and glass can creat highest shock pressure compared with other CMs. The figure has been drwan by carrying out of equations 1 to 5 . The figure 5 indicates influence of PC on shock pressure.

This figure shows that the maximum resulted shock pressure can be generated with beryllium foil as PC. Beryllium with the highest sound speed $(12800 \mathrm{~m} / \mathrm{s})$, provide higher $\mathrm{Z}$ and according to eqaution 1 , the shock pressure can be increased. Furthermore, lower density of beryllium as PC compared with specimen (magnesium) leads enhancement of shock pressure, as well. This phenomenon can be interpreted by mistmached theory in equation 9 [11].

$\frac{P_{2}}{P_{1}}=\frac{4 \rho_{2}}{\left[\left(\rho_{2}\right)^{\frac{1}{2}}+\left(\rho_{1}\right)^{\frac{1}{2}}\right]^{2}}$

Where $\mathrm{P}_{1}$ is shock pressure before the protective coating that is calculated by equation $1, \rho_{1}$ is density of $\mathrm{PC}, \rho_{2}$ is density of specimen and $\mathrm{P}_{2}$ is resulted pressure. the figure 5 has been achieved by equations 1 to 5 and 9. More elaborate explanations, have been proposed in recent research of this manuscript authors [12]

Even though beryllium can amplify the shock pressure, considering the cost of $\mathrm{Be}, \mathrm{PC}$ has not been used in our experiments.

\section{Design of Experiments (DOE)}

In design of experiments, the significant parameters should be cosidered as involved factors. The equation 3 indicates the laser intensity (I) is one of important parameters which is varied by beam diameter (D). It must be noted that repetition rate, laser power and pulse duration have been assumed as constant parameters. In addition to D, number of scans ( the number of repeating shots at an identical point) and percentage of overlap are other variables in this work. Since, some portion of laser energy is absorbed by through passing of optical devices, the effective laser power should be determined. As shown in figure 6, The laser power in A, B, and $\mathrm{C}$ is measured 
by Gentec $U N O$ at $4.8 \mathrm{~W}, 3.55 \mathrm{~W}$ and $3.52 \mathrm{~W}$, respectively.

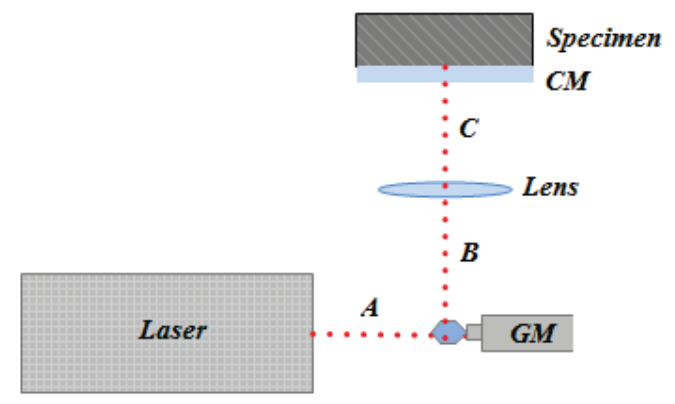

Fig.6: Measurement average laser power in different regions

By having, effective laser power (3.52 $\mathrm{W}$ at $\mathrm{C})$ and conduction parameters of table 1at equations 1-5, the proper $\mathrm{D}$ for laser peening must be less than $70 \mu \mathrm{m}$. Furthermore, some constraints in applied optical devices leads more reduction of D (less than $35 \mu \mathrm{m}$ ) cannot be executable. Thus, two magnitudes of $\mathrm{D}$ at 40 and $60 \mu \mathrm{m}$ are used as proper choices.

In all experiments the used laser is PRISMA TM 1064-V diode pumped, solid-state laser. The laser power is $3.52 \mathrm{~W}$ at $10 \mathrm{KHz}$ repetition rate. Pulse duration is $14 \mathrm{~ns}$, wavelength is $1064 \mathrm{~nm}$ and initial beam diameter (D) varied from 5.06 to $3.37 \mathrm{~mm}$ for 40 and $60 \mu \mathrm{m}$ spot sizes, respectively. Therefore, the intensity of $2 \mathrm{GW} / \mathrm{cm}^{2}$ at $40 \mu \mathrm{m}$ spot size and 0.91 $\mathrm{GW} / \mathrm{cm}^{2}$ at $60 \mu \mathrm{m}$ spotsize is available for peening magnesium specimens, where glass with $1 \mathrm{~mm}$ as CM are clamped to specimens. To have grater effect of shock pressure, the interface between $\mathrm{CM}$ and surface of specimen should be reduced as possible, otherwise air can be considered as CM instead of glass and shock pressure declines, drastically. To reduction thickness of interface between $\mathrm{CM}$ and specimen, all specimens have been polished to mirror surface and surface roughness has been reduced to $0.25 \mu \mathrm{m}$. Considering the two variables for $\mathrm{D}$ (40 and 60 $\mu \mathrm{m})$, three variables for number of scans $(1,2$ and 3 scans) and three variables for \%overlap (0\%, 33\% and 66\%), 18 experiments must be performed. Hence, eighteen circular specimens $(99.8 \% \mathrm{Mg}-0.2 \% \mathrm{Ca})$ with $33 \mathrm{~mm}$ diameter and $6 \mathrm{~mm}$ thickness have been prepared for experiments as in table2. In the first three experiments 1-3, the spot size is maintained at $40 \mu \mathrm{m}$, peening overlap at $0 \%$ and the effect of number of scans 1,2, 3 are studied. In experiments $4-9$ the overlap was increased to $33 \%$ and $66 \%$ by varying the scan speed, as shown in figure 1 . In the next 9 experiments, 10-18, the spot size is changed to $60 \mu \mathrm{m}$ and the effect of reduction in intensity by increasing spot size diameter, percentage of overlap and number of scans are studied. In order to evaluate the occurrence of HRLSP on magnesium, three approaches are proposed in this research.

a) Measurement depth of deformed rigions upon the specimen surface by WYKO NT1100 interfrometer and comparison of theoretical and experimental magnitudes. b) Measurement increasing hardness by $M V K-H 1$ Mitutoyo micro-hadness tester.

c) Measurement of changed surface roughness by $W Y K O$ NT1100 interfrometer and scanning electron microscopy (SEM).

Table 2-List of experiments

\begin{tabular}{|c|c|c|c|}
\hline $\begin{array}{c}\text { Specimen } \\
\text { number }\end{array}$ & $\begin{array}{c}\text { Spot } \\
\text { size(D) }\end{array}$ & \%Overlap & $\begin{array}{c}\text { Number } \\
\text { of scans }\end{array}$ \\
\hline 1 & 40 & 0 & 1 \\
\hline 2 & 40 & 0 & 2 \\
\hline 3 & 40 & 0 & 3 \\
\hline 4 & 40 & 33 & 1 \\
\hline 5 & 40 & 33 & 2 \\
\hline 6 & 40 & 33 & 3 \\
\hline 7 & 40 & 66 & 1 \\
\hline 8 & 40 & 66 & 2 \\
\hline 9 & 40 & 66 & 3 \\
\hline 10 & 60 & 0 & 1 \\
\hline 11 & 60 & 0 & 2 \\
\hline 12 & 60 & 0 & 3 \\
\hline 13 & 60 & 33 & 1 \\
\hline 14 & 60 & 33 & 2 \\
\hline 15 & 60 & 33 & 3 \\
\hline 16 & 60 & 66 & 1 \\
\hline 17 & 60 & 66 & 2 \\
\hline 18 & 60 & 66 & 3 \\
\hline
\end{tabular}

\section{Results and Discussion}

\subsection{Peening depth evaluation}

Plastic deformation by laser peening leads to change in surface topography. This issue has been presented by previous research on magnesium [13].

Measurement of depth of peened regions and comparison with theoretical magnitude that can be calculated by equation 10 is one of the reliable methods for assessing HRLSP occurrence [10].

$\varepsilon_{\mathrm{P}}=-\frac{2 \mathrm{HEL}}{2 \mu+3 \lambda}\left(\frac{\mathrm{P}}{\mathrm{HEL}}-1\right)$

Where, $\varepsilon_{\mathrm{P}}$ is depth of peening due to one laser shot, $\mathrm{P}$ is shock shock pressure, $\mu$ and $\lambda$ are lama constants those are 15.4 and $36.3 \mathrm{GPa}$ respectively for magnesium.

Figures 7 and 8, demonstrate the interfrometric line scan of samples peened with 40 and $60 \mu \mathrm{m}$ laser spots in a single scanning pass and $0 \%$ overlap. In the figures the $\mathrm{x}$ axis shows the width of the peened spots and the y axis shows the depth of peening. Also the theoritical depth of peening calculated by equation 10, is shown as reference in the figure with a broken line $(3.97 \mu \mathrm{m}$ for $40 \mu \mathrm{m}$ spot and $2.24 \mu \mathrm{m}$ for $60 \mu \mathrm{m}$ spot). 


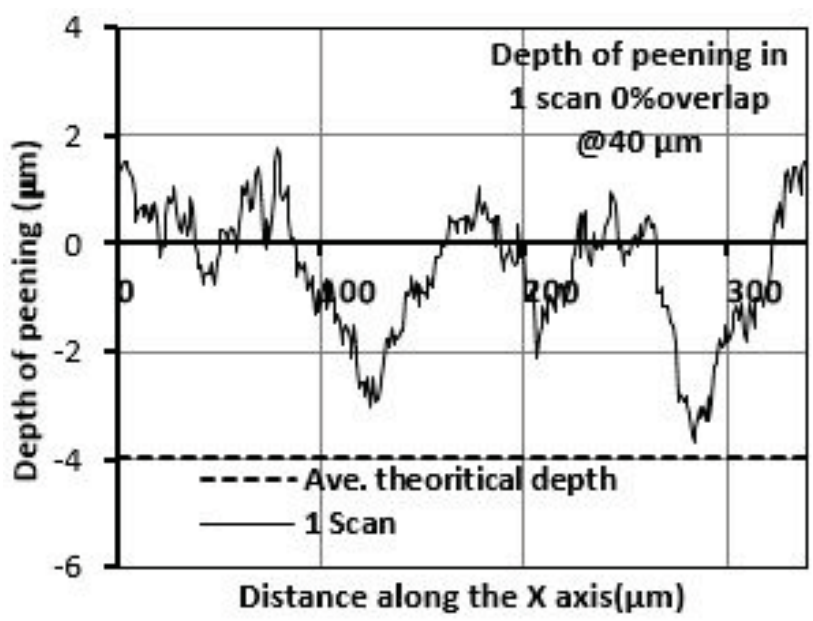

Fig.7-Fluctuations of peening depth at $40 \mu \mathrm{m}$

It can be clearly seen that magnitude of peened depth 3 to $3.8 \mu \mathrm{m}$ from experiments is close to the theoritical magnitude with $3.97 \mu \mathrm{m}$. As the spot size increases to $60 \mu \mathrm{m}$, this difference becomes $2 \mu \mathrm{m}$ in figure 8 .

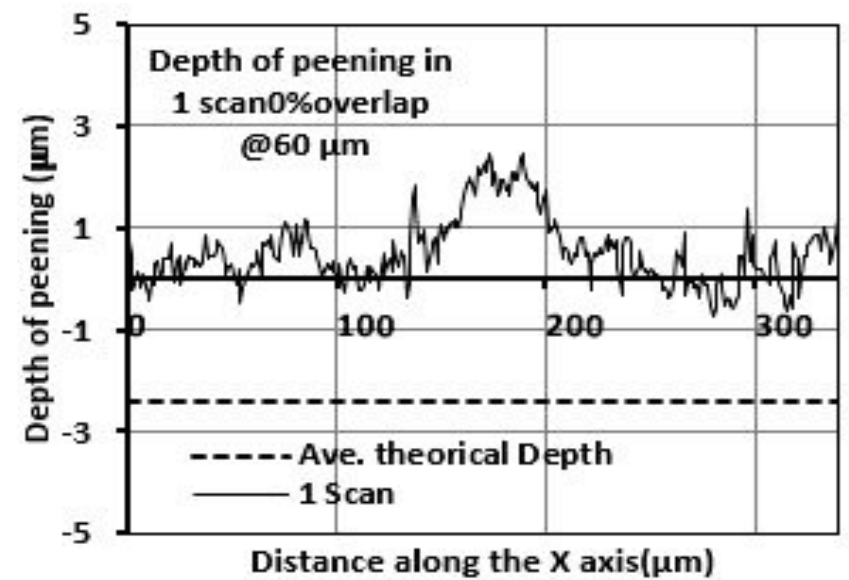

Fig.8-Fluctuations of peening depth at $60 \mu \mathrm{m}$

The intensity has been reduced to $0.91 \mathrm{GW} / \mathrm{cm}^{2}$. The notable difference in experimental and theoritical results at $60 \mu \mathrm{m}$ spot size is caused by reduction in laser intensity, which is not sufficient for effective LP. Regarding $60 \mu \mathrm{m}$ experiments, it can state there is a combination of peening and machining (surface melting). Furthermore, in both figures theoritical depth in all experiments are more than experimental results. Although, the measured laser power before the $\mathrm{CM}$ is $3.52 \mathrm{~W}$, hot plasma region reduces the CM transparancy. This means additional reduction in laser power after CM could be occurred and the real laser intensity is less than theory. In addition, oxidation of magnesium, forming a $5 \mu \mathrm{m}$ thick layer [14] increases the hardness of magnesium which also increases the required pressure for peening. Therby, reducing the peening depth. This has been verified by conduction of micro hardness test immediately after polishing and the specimen that was used in experiment which shows increase in hardness from 38 to $45 \mathrm{HV}$ due to oxidation. Though aforementioned reasons lead to reduction in shock pressure, the rest of the laser power has been adequate for peening at $40 \mu \mathrm{m}$ spot size.

\subsection{Hardness}

Plastic deformation upon the specimen surface increases CRS [15]. The relation of hardness and CRS on magnesium has been observed in the literature [13]. Hence, notable change in hardness can be considered as an evidence to occurrence of HRLSP. Increasing laser intensity causes increase in plasma pressure resulting in higher CRS and hardness. The effect of scanning parameters (percentage of overlap and number of scans) on hardness can be realized in figures 9 and 10, where the $\mathrm{X}$-axis indicates \% overlap and Y-axis specifies Vickers Hardness for various number of scans compared with unpeened specimens. In figure 9, it can be clearly seen that the hardness increases from $45 \mathrm{HV}$ for unpeened sample to $60 \mathrm{HV}$ for single scan at $0 \%$ overlap. This significant increase in hardness at single scan and $0 \%$ overlap can show the occurrence of laser peening. Note that, heat treatment (by laser heating) cannot be effective for enhancement of pure magnesium and it can be hardened by strain hardening that is created by cold working in laser shock peening [16]. Furthermore, figure 9 demonstrates increase in number of scans and percentage of overlap leading to additional increase in hardness. This is similar to another reported work on laser peening [6]. From figure 9, the maximum achieved hardness is $103 \mathrm{HV}$ that is 2.5 times higher than unpeened specimen. The comparable result has been reported in recent works on magnesium [13]. The figure 9 indicates that the hardness of 3 scans, $0 \%$ overlap is $74 \mathrm{HV}$ which is close to hardness of $71 \mathrm{HV}$ at one scan, $66 \%$ overlap. This is due to equal energy magnitude has been applied to the identical area of specimens by the same numbers of laser shots. This shows that, the increase in hardness is correlated to the magnitude of applied energy used for laser peening. Figure 10 demonstrates slight increase the hardness from 45

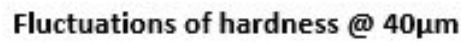

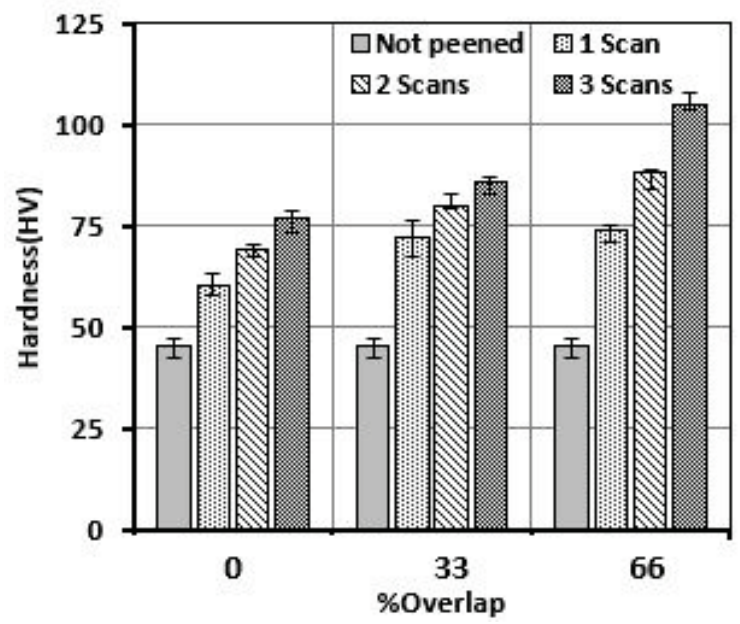

Fig.9- Fluctuations of hardness @ $40 \mu \mathrm{m}$ 




Fig.10: Fluctuations of hardness @ $60 \mu \mathrm{m}$

$\mathrm{HV}$ for unpeened specimen to $51 \mathrm{HV}$ for single scan at $0 \%$ overlap. Additionally, increase in number of scans and percentage of overlap has a negligible effect, where the maximum achieved hardness is $59 \mathrm{HV}$ that is just 1.25 times higher than unpeened specimen. Referring figure 4 , it can be seen that the increase in spot size from 40 to $60 \mu \mathrm{m}$, causes reduction in shock pressure. Consequently, the inferior shock pressure cannot create prominent cold working at $60 \mu \mathrm{m}$ and lower hardness can be resulted. Therefore, even by increasing of number of scan and \%overlap, enhancement of hardness in $60 \mu \mathrm{m}$ experiments is not impressive.

\subsection{Surface topography and roughness}

A number of researches indicate that the controlled periodic shock pressure create uniform micro plastic deformation on the specimen surface [13][17]. Figures 11 and 12 show SEM of the specimen surface after HRLSP at 40 and $60 \mu \mathrm{m}$ spot sizes, respectively (enlarged image shown as insert in the images for clarity). At $40 \mu \mathrm{m}$, there is no melting and solidified material remains on the peened region and the uniform surface is produced after laser irradiation, which can be seen in the SEM scan in figure 11. In lower laser intensity at $60 \mu \mathrm{m}$, it cannot see the same arrangement on the surface. The lower laser intensity cannot provide required energy to ablation and combination of LSP and laser surface melting (LSM) is created as shown in figure 12. Note that, in LSM,

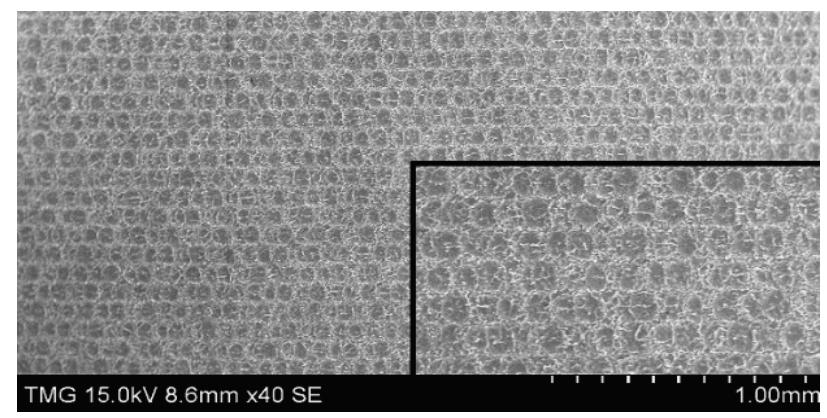

Fig.11: HRLSP of magnesium surface $@ 40 \mu \mathrm{m}$ spot size the laser energy is just adequate for converting surface specimen from solid to liquid stage. Closer inspection of figure 12 , shows that the depth is shallower compared to peening at $40 \mu \mathrm{m}$ and machined holes at the center of the

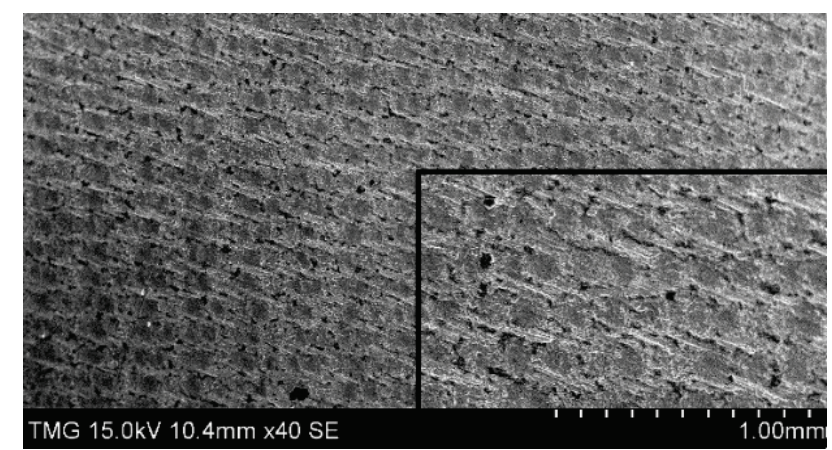

Fig.12: HRLSP of magnesium surface @ 60 $\mu \mathrm{m}$ spot size

spots could be observed. The melting that happens while machining causes solidified material to settle in the processed region which creates some disarrangement. In order to more study the surface, average roughness $\left(\mathrm{R}_{\mathrm{a}}\right)$ of all 18 specimens was measured with WYKO NT1100 interfrometer. The results of these measurements are shown in figures 13 and 14 where

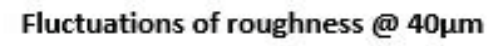

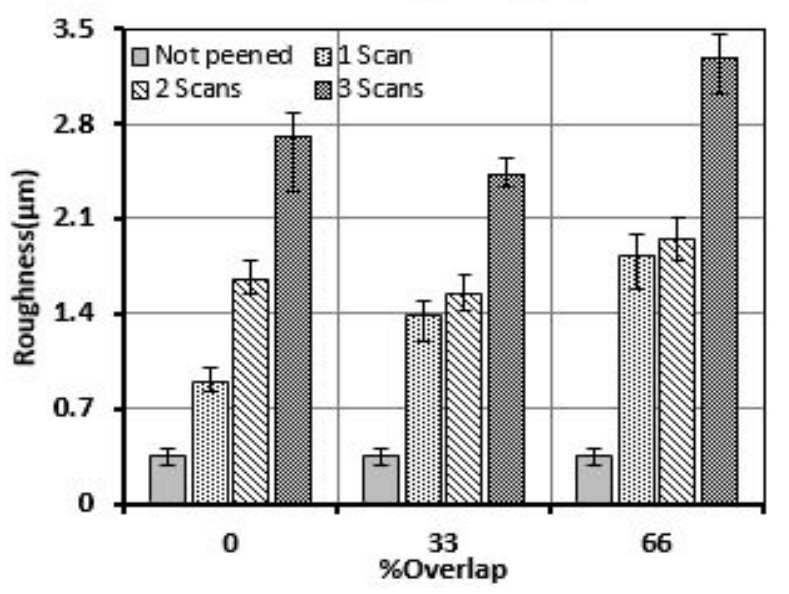

Fig.13: Fluctuations of roughness @ $40 \mu \mathrm{m}$

the $\mathrm{X}$-axis specify \%overlap and $\mathrm{Y}$-axis indicate the $\mathrm{R}_{\mathrm{a}}$ value. In figure 13 , it can be clearly seen that $R_{a}$ increases with increase in number of scans. For instance in $0 \%$ overlap peening, the roughness has an important enhancement from 0.35 (not peened) up to $2.6 \mu \mathrm{m}$ (3 scans). Correspondingly, for $33 \%$ overlap, the $\mathrm{R}_{\mathrm{a}}$ enhances from 0.35 to $1.85 \mu \mathrm{m}$ and for $66 \%$ overlap, it increases from 0.35 to $3.25 \mu \mathrm{m}$. It can also be seen that the $\mathrm{R}_{\mathrm{a}}$ increases with increase in percentage of overlap, when using single scan, from $0.8 \mu \mathrm{m}$ (single scan $0 \%$ overlap) to $1.8 \mu \mathrm{m}$ (single scan $66 \%$ overlap). However, at multiple scans peening there is a reduction while the overlap has been $33 \%$. Increasing the $\%$ overlap eliminates some peaks from the previous scan. However, at higher overlap of $66 \%$ further plasticized material is being driven to peening sides and $R_{a}$ increases. In figure 14 , the increase in 


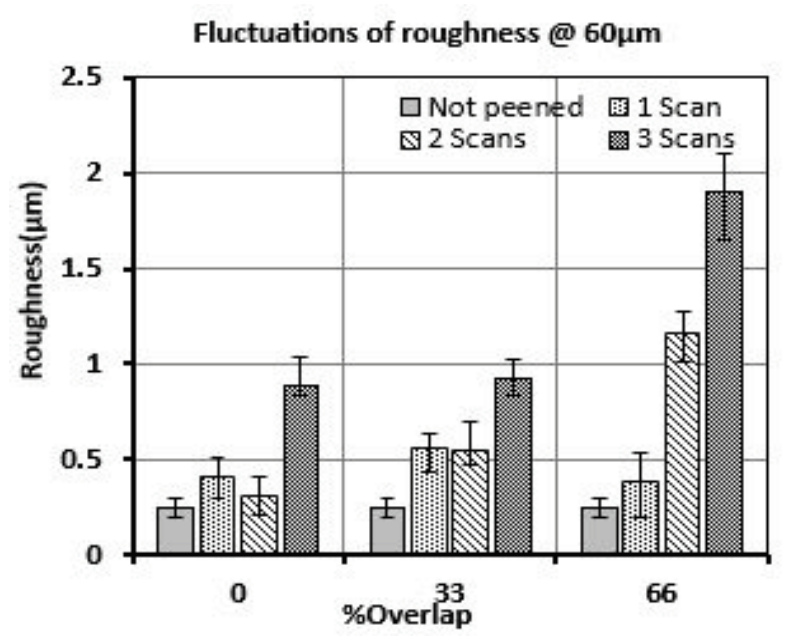

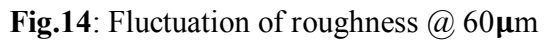

$\mathrm{R}_{\mathrm{a}}$ is marginal for single scan regardless of the \%overlap at $60 \mu \mathrm{m}$. The $\mathrm{R}_{\mathrm{a}}$ increases from 0.35 to $1 \mu \mathrm{m}$ in case of $66 \%$ overlap and 2 scans. Highest increase in $\mathrm{R}_{\mathrm{a}}$ from 0.35 to $1.8 \mu \mathrm{m}$ occurs at 3 scans, $66 \%$ overlap peening. At $66 \%$ overlap, 3 scans specimen this solidified material causes notable increase in $\mathrm{R}_{\mathrm{a}}$.

\section{Conclusion:}

In this work, high repetition rate laser shock peening of magnesium is performed. To knowledge of the authors, this work is the first HRLSP on magnesium. The $\mathrm{NdYVO}_{4}$ laser used for the experiments has a power of $3.52 \mathrm{~W}$ at $10 \mathrm{KHz}$ repetition rate. The success of peening was evaluated by measuring depth of peening, change in micro hardness and surface roughness of peened samples in comparison to unpeened magnesium. Though at $60 \mu \mathrm{m}$ spot size $(\mathrm{I}=0.91$ $\mathrm{GA} / \mathrm{cm}^{2}$ ), the theoretical shock pressure is higher than the HEL of magnesium, evaluation of peening depth, hardness, and $R_{a}$ show that there is no appreciable increase in any of these parameters when compared with unpeened magnesium. Furthermore, the SEM images clearly show evidence of machining and solidified material at $60 \mu \mathrm{m}$. However, at 40 $\mu \mathrm{m}$ laser spot size $\left(\mathrm{I}=2 \mathrm{GW} / \mathrm{Cm}^{2}\right)$ there is appreciable increase in hardness, and $\mathrm{R}_{\mathrm{a}}$ when compared with unpeened magnesium. This evidence shows effective laser peening at $40 \mu \mathrm{m}$. At one scan, $0 \%$ overlap, the depth of peening is calculated to be $3.97 \mu \mathrm{m}$ and the measured depth is $3.8 \mu \mathrm{m}$ which is very close to theoretical value. The surface hardness increased from 45 to $103 \mathrm{HV}$ which is very similar to the hardness achieved by other works on laser peening of magnesium. In addition, appreciable increase in $\mathrm{R}_{\mathrm{a}}$ from 0.35 $\mu \mathrm{m}$ for unpeened specimen to $3.3 \mu \mathrm{m}$ for peened specimen with a $40 \mu \mathrm{m}$ spot size. Also the SEM images clearly show evidence of uniform peening without any trace of machining or solidified material at this intensity. Hence, the occurrence of HRLSP could be could be proved at $40 \mu \mathrm{m}$.

\section{References}

[1] M. Salahshoor, and Y. Guo:J. Materials (Basel).,5, (2012) 135.

[2] C. Ruff, B. Holt, and E. Trinkaus: J.Wolff s Law and Bone Functional Adaptation., 498, (2006) 484.

[3] G. Song: J.Corros. Sci., 49, (2007) 1696.

[4] X. C. Zhang, Y. K. Zhang, J. Z. Lu, F. Z. Xuan, Z. D. Wang, and S. T. Tu: J.Mater. Sci. Eng. A., 527, (2010) 3411.

[5] P. Peyre, X. Scherpereel, L. Berthe, C. Carboni, R. Fabbro, G. Béranger, and C. Lemaitre: J.Mater. Sci. Eng. A, 280, (2000) 294.

[6] H. P. L. b Peyre a R. Fabbro , P. Merrien:J. Mater. Sci. Eng., 210, (1996)102.

[7] H. S. Niehoff, F. Vollertsen, and B. Institut: J. Metal., $110,(2007) 110$.

[8] K. D. and L.ye:"Laser shock peening performance and process simulation." (woodhead publishing limited, UK, 2006) P.17.

[9] W. Braisted and R. Brockman: J.Int. J. Fatigue., 21, (1999)719.

[10]A. Kruusing, "Handbook of Liquids-Assisted Laser Processing."( Elsevier, US, 2007)P.98.

[11] M. Shukla, Y. Kashyap, P. . Sarkar, a Sinha, H. . Pant, R. . Rao, N. . Gupta, V. . Senecha, and B. . Godwal: J. Nucl. Fusion., 46, (2006)419.

[12] H. Kamkarrad, S. Narayanswamy, and X. S. Tao: J. Int. J. Adv. Manuf. Technol., 74, (2014)1237.

[13]Y. Guo, M. P. Sealy, and C. Guo: J. CIRP Ann. Manuf. Technol., 61, (2012)583.

[14] J. Yang, F. Cui, and I. S. Lee: J. Ann. Biomed. Eng., 39, (2011)1857.

[15]M. Turski, S. Clitheroe, A. D. Evans, C. Rodopoulos, D. J. Hughes, and P. J. Withers: J. Appl. Phys. A.,99, (2010)549.

[16]C. H. Caceres and P. Lukac: J. Philos. Mag., 88, (2008)977.

[17] M. P. Sealy and Y. B. Guo: J. Mech. Behav. Biomed. Mater., 3, (2010)488.

(Received: July 16, 2015, Accepted: November 3, 2015) 